\title{
(2) OPEN ACCESS \\ Predicting inhospital admission at the emergency department: a systematic review
}

\author{
Anniek Brink 이, ', Jelmer Alsma 다, 'Lodewijk AAM van Attekum, ${ }^{1}$ \\ Wichor M Bramer, ${ }^{2}$ Robert Zietse, ${ }_{1}^{1}$ Hester Lingsma, ${ }^{3}$ Stephanie CE Schuit ${ }^{1}$
}

\section{Handling editor Kirsty Challen}

- Additional supplemental material is published online only. To view, please visit the journal online (http://dx.doi. org/10.1136/emermed-2020210902).

'Department of Internal Medicine, Erasmus MC, Rotterdam, The Netherlands ${ }^{2}$ Medical Library, Erasmus MC, Rotterdam, The Netherlands ${ }^{3}$ Public Health, Erasmus MC, Rotterdam, The Netherlands

\section{Correspondence to}

Anniek Brink, Department of Internal Medicine, Erasmus MC, Rotterdam, Zuid-Holland, The Netherlands;

a.brink@erasmusmc.nl

Received 16 November 2020 Accepted 8 October 2021

\section{Check for updates}

(c) Author(s) (or their employer(s)) 2021. Re-use permitted under CC BY. Published by BMJ.

To cite: Brink A, Alsma J van Attekum LAAM, et al. Emerg Med I Epub ahead of print: [please include Day Month Year]. doi:10.1136/ emermed-2020-210902

\section{ABSTRACT}

Background ED crowding has potential detrimental consequences for both patient care and staff. Advancing disposition can reduce crowding. This may be achieved by using prediction models for admission. This systematic review aims to present an overview of prediction models for admission at the ED. Furthermore, we aimed to identify the best prediction tool based on its performance, validation, calibration and clinical usability. Methods We included observational studies published in Embase.com, Medline Ovid, Cochrane CENTRAL, Web of Science Core Collection or Google scholar, in which admission models were developed or validated in a general medical population in European EDs including the UK. We used the Critical Appraisal and Data Extraction for Systematic Reviews of Prediction Modelling Studies (CHARMS) checklist to assess quality of model development. Model performance was presented as discrimination and calibration. The search was performed on 11 October 2020.

Results In total, 18539 articles were identified. We included 11 studies, describing 16 different models, comprising the development of 9 models and 12 external validations of 11 models. The risk of bias of the development studies was considered low to medium. Discrimination, as represented by the area under the curve ranged from 0.630 to 0.878 . Calibration was assessed in seven models and was strong. The best performing models are the models of Lucke et al and Cameron et al. These models combine clinical applicability, by inclusion of readily available parameters, and appropriate discrimination, calibration and validation.

Conclusion None of the models are yet implemented in EDs. Further research is needed to assess the applicability and implementation of the best performing models in the ED.

Systematic review registration number PROSPERO CRD42017057975.

\section{INTRODUCTION}

It is of great importance to provide timely care for patients in the ED. However, sometimes this is compromised by ED crowding, a situation that occurs when there are more patients than available beds in the ED. ${ }^{1}$ ED crowding has direct and indirect detrimental consequences for patient care and ED staff. ${ }^{2}$ It leads to an increase in the length of stay (LOS) at the ED, a longer inhospital LOS and an increase in morbidity and mortality. ${ }^{4-7}$ There are several causes proposed for the emergence of ED crowding. Asplin et $a l^{8}$ introduced a conceptual

\section{Key messages}

What is already known on this subject

- Several admission prediction tools have been developed with the intention to shorten length of stay at the ED in an attempt to reduce crowding. Implementation of a tool into every day practice has not yet occurred, as this can only be done after validation and calibration in the hospital where it is going to be used. No research to evaluate and compare these models has been published yet.

\section{What this study adds}

- This systematic review is the first to critically appraise these admission prediction tools. Of the 16 models that we reviewed, only few were adequately developed, validated and calibrated.

model of ED crowding, visualising the factors associated with crowding. These factors can be divided into input, throughput and output factors. It is thought that mainly output, that is, an inadequate disposition of patients, contributes to crowding, which subsequently leads to limited patient flow at the ED. Especially elderly are at risk for a long LOS and many need to be admitted. ${ }^{9}{ }^{10}$ Advancing patient disposition may reduce LOS at the ED and thus consequently reduce crowding. The identification of those patients that need admission at ED arrival may help to shorten ED LOS for many patients. Earlier admission (ie, shorter time in the ED) is associated with improved patient outcomes. ${ }^{11}$ Several prediction tools exist to identify patients needing hospital admission. Implementing such a model in clinical practice may alter patient courses and lead to earlier admission. ${ }^{12}$ However, a clear overview of literature concerning admission models has not yet been presented. Therefore, the aim of this systematic review is to give an overview of present knowledge on admission prediction models in a general ED population. The secondary aim was to assess the quality of the developed prediction models. As many studies targeted the older population, we will also provide an overview of prediction models developed for this population.

\section{METHODS}

The study was conducted and reported according to the Preferred Reporting Items for Systematic Reviews and Meta-Analyses (PRISMA) guidelines. ${ }^{13}$ We performed a systematic review on prediction 
models on admission in the ED. The study protocol is registered in the International Prospective Register of Systematic Reviews (PROSPERO) under registration number CRD42017057975.

\section{Eligibility criteria}

We aimed to identify all models developed until 11 October 2020 for a non-trauma ED population or that were applicable to a mixed trauma and non-trauma population. The articles needed to fulfil the following criteria to be considered for inclusion: (1) the prediction tool was developed or validated in an adult population, (2) the prediction model did not have predefined illnesses (eg, pneumonia) or symptoms (eg, tachycardia) as inclusion criteria, and (3) the article described a model rather than only individual predictors. Studies that concerned case reports, reviews or meta-analyses were excluded. Moreover, the search was restricted to articles written in English. The references of eligible studies were analysed to identify additional articles for inclusion. Because of the heterogeneity between ED systems worldwide, we limited our search to prediction models developed or validated in European EDs including the UK.

During our initial assessment of the literature, we found multiple models for the elderly population. Therefore, we also decided to give an overview of this subgroup of models.

\section{Information sources}

The following databases were searched from inception until 11 October 2020: Embase.com, Medline ALL via Ovid, Cochrane CENTRAL Register of Trials via Wiley, Web of Science Core Collection and Google Scholar.

\section{Search}

We used among others the following keywords: prediction, risk, hospital admission, emergency department, model and related synonyms. The queries were developed in Embase.com, and syntax and thesaurus terms were afterwards adjusted for the other databases. The search strategy was created by a biomedical information specialist (WMB). See online supplemental appendix A for the complete syntaxes.

\section{Study selection}

Duplicate articles were removed using Endnote for Windows (Thomson Reuters, V.X9) using the method as described by Bramer et al. ${ }^{14}$ Two researchers (AB and LAAMvA) independently performed the screening of title and abstracts. Conflicting results were discussed in consensus meetings. After screening the abstracts, the full text of the articles was assessed for eligibility by the same researchers and included or excluded in the systematic review. Any remaining disagreement between the first two researchers was discussed with a third investigator (JA).

\section{Data collection process $\&$ data items}

The following data were extracted from every included article: year of publication, author, journal of publication, country of the study, study period, study design, inclusion and exclusion criteria in their study, study population, hospital setting (ie, regional hospital, tertiary care hospital), outcome (ie, number of admissions), model name, parameters within the model, model performance (eg, discrimination and calibration), sample size, derivation and/or validation study, calibration method, handling of missing data and patient characteristics (ie, age, sex). Data were extracted by one investigator $(\mathrm{AB})$ and a random check was performed by a second investigator (JA). This check showed no discrepancies.

\section{Risk of bias in individual studies}

The methodological quality and the risk of bias were assessed with the Critical Appraisal and Data Extraction for Systematic Reviews of Prediction Modelling Studies (CHARMS) checklist, which can be used to describe the reliability, applicability and reproducibility of prediction models. ${ }^{15}$ This checklist is applicable for studies deriving a prediction model. The CHARMS checklist assesses risks for bias in the following areas: participant selection, predictor assessment, outcome assessment, model development and analysis. The results were aggregated into a low, moderate or high risk of bias. The risk of bias was assessed by two investigators ( $\mathrm{AB}$ and LAAMvA). Discrepancies were discussed with a third investigator (JA).

\section{Summary measures, data synthesis and analysis}

We evaluated the predictive performance of the models described in terms of discrimination and calibration. Discrimination is a measure of how well a model can distinguish the high-risk from the low-risk group for a certain outcome. It is usually presented as an area under the curve (AUC) in which a value closer to 1.0 indicates better distinction. ${ }^{16}$

Calibration reflects the agreement between the expected (ie, predicted) and observed outcome. This can either be assessed by the Hosmer-Lemeshow goodness of fit test, calibration curve, Wilcoxon signed rank test, Schwarz Bayesian Information Criterion or Brier score. ${ }^{17}$ The Hosmer-Lemeshow assesses whether the expected event numbers match the observed event numbers. It provides a $\chi^{2}$ statistic and accessory $\mathrm{p}$ value. A non-significant $\mathrm{p}$ value indicates good calibration. The calibration curve contains a slope and intercept, in which the intercept reflects whether predictions are systematically too low or too high. The calibration slope is a reflection of the predictor effects within the model. ${ }^{17}$ The Wilcoxon signed rank test compares two datasets which are not normally distributed. A significant $\mathrm{p}$ value implies that a prediction model performs different in the separate datasets. If a study reported multiple prognostic models or multiple stages of prognostic modelling (eg, development and validation), data extraction was performed separately for each model or stage. We classified prognostic models as separate models when they included a different set of prognostic variables. Models with identical predictors but for different outcomes were considered validation studies.

Patient characteristics were presented as mean with SD, median with IQR or numbers with percentages dependent on the distribution of the data. As a result of the heterogeneity of the patient population and the prediction models, a metaanalysis was not possible.

\section{RESULTS}

\section{Study selection}

In the literature search, we detected 18539 studies, of which 13017 remained after deduplication. The exclusion of studies based on title and abstract resulted in 104 full text articles eligible for detailed assessment. The main reasons for excluding articles were that the study described was performed in non-European EDs $(n=29)$ or that different outcomes were studied (eg, revisits or LOS) $(n=29)$. Finally, we included 11 articles in this systematic review. Full details of study selection are summarised in figure 1. 


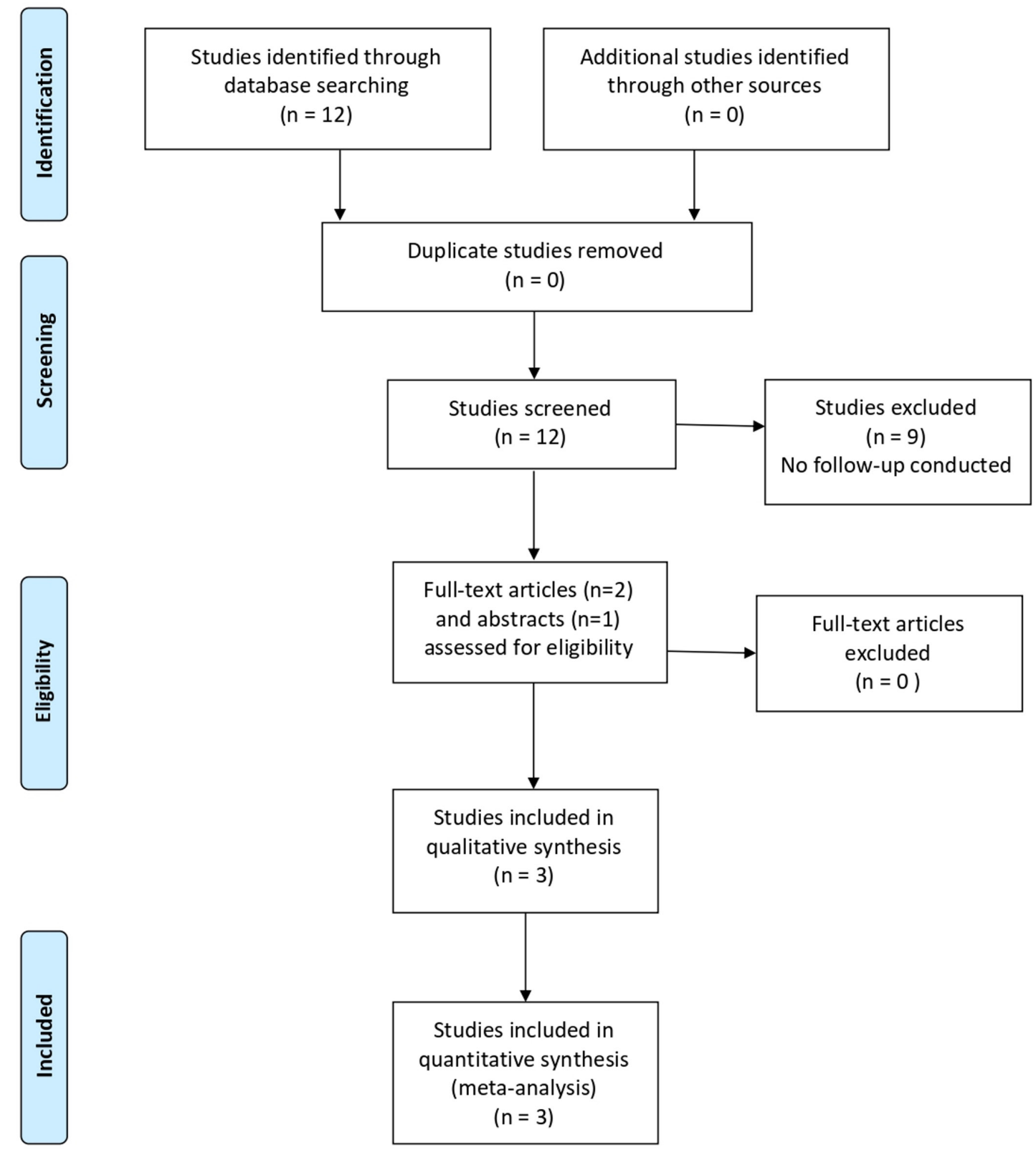

Figure 1 Flowchart for literature search on prediction models for admission.

\section{Study characteristics}

Study characteristics are summarised in table 1 . The 11 studies described 16 different models. Two models were tested in two different studies (Identification of Seniors At Risk (ISAR) and Glasgow Admission Prediction Score (GAPS)). ${ }^{18-21}$ Most models were constructed with logistic regression. Only three models were developed using machine learning. ${ }^{22} 23$

Seven included studies had a prospective design and the majority of the studies were carried out in a single centre $(n=8)$. None of the studies assessed prospectively the performance of the model when implemented in day to day practice.

\section{Quality assessment}

The quality of the studies in which a model was developed $(n=5)$ was assessed using the CHARMS checklist. ${ }^{19}$ 22-25 This considered five studies in which nine models were developed. The results of the CHARMS were aggregated into low, medium and high risk for bias (table 2) (online supplemental appendix C).
The risk of bias is evaluated with the CHARMS checklist, which assesses the domains of participant selection, predictor assessment, outcome assessment, model development and analysis. The results are summarised as low (L) risk of bias, moderate (M) risk of bias or high $(\mathrm{H})$ risk of bias.

The study attrition, referring to the method in which patients were recruited for inclusion, was of good quality in all studies. However, two studies did not describe basic patient characteristics. ${ }^{19} 23$ The outcome was described in all studies. Since the prediction tool had to predict an event in the near future (ie, admission from the ED), loss to follow-up was considered as non-important. Furthermore, the number of patients who were transferred to other hospitals or who left without being seen did not exceed $20 \%$. The number of outcomes in all studies was described and therefore also the number of candidate predictors was satisfying. However, just one study explicitly mentioned that they took into account the number of events per variable to limit overfitting of the model. ${ }^{25}$ In general, the number of events 




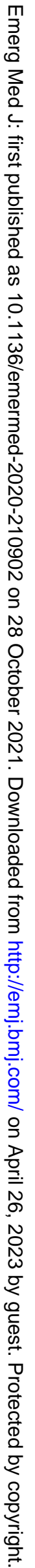


Table 2 Risk of bias in the development studies

\begin{tabular}{|c|c|c|c|c|c|}
\hline & Cameron et al ${ }^{19}$ & Kraaijvanger et al ${ }^{24}$ & Lucke et $a l^{25}$ & Noel et $a l^{22}$ & Zlotnik et all ${ }^{23}$ \\
\hline Participant selection & $\mathrm{L}$ & $\mathrm{L}$ & L & $\mathrm{L}$ & $\mathrm{L}$ \\
\hline Predictor assessment & L & L & L & $M$ & L \\
\hline Outcome assessment & L & L & L & L & L \\
\hline Model development & L & $M$ & L & M & M \\
\hline Analysis & L & L & L & $M$ & L \\
\hline
\end{tabular}

$\mathrm{L}$, low risk of bias; $\mathrm{M}$, moderate risk of bias.

per variable should at least be 10 , meaning that if 100 events happened the maximum number of predictors in a model is 10 .

All studies included parameters that are easily obtainable during triage. Furthermore, one study provided two models which included the triage nurse prediction on admission. ${ }^{22}$ This is a subjective parameter and therefore difficult to reproduce. However, in the third model by Noel et al the triage nurse prediction was not included.

The majority of the models (13/16) were developed using logistic regression, but in three automated computer techniques were used. $^{22} 23$ All studies used age as a categorical variable in the model. However, it is not clearly described whether categorisation of parameters took place before or after inclusion in multivariable analysis.

Description of missing data and handling of missing data were not available for every study, ${ }^{23} 24$ one study excluded patients with missing values ${ }^{22}$ and two studies compensated missing values. $^{19} 25$

External validation is considered to be the best validation method. Two studies performed external validation, ${ }^{24} 25$ while two others used internal validation. ${ }^{19} 23$ One study did not perform validation and was therefore considered a high risk of bias. $^{22}$

Overall, the models of Cameron et $a l^{19}$ and Lucke et $a l^{25}$ were considered to be developed best with an on average low risk on bias in the CHARMS checklist.

\section{Participant characteristics}

Population size ranged from 274 to 322846 patients and contribution of male patients ranged from $39.0 \%$ to $54.7 \%$. Mean age (SD) ranged from $41(22)$ to 84 (5.5) years. Four studies included older ED patients, defined as either $\geq 65$ years $^{21} 2627$ or $\geq 75$ years. $^{20}$ One study compared the older ED population (age $\geq 70$ years) with the general adult population. ${ }^{25}$

\section{Outcome characteristics}

Admission rates varied from $13.6 \%$ in adults to $59.4 \%$ in the older patient population.

\section{Variables included in the scoring systems}

The number of parameters ranged from 1 aggregated score (ie, Emergency Severity Index (ESI)) to 13 parameters. We subsequently categorised these parameters into demographics, vital signs, interventions, triage, previous care contacts, chief complaint, drug use, mobility and dependency, ED entrance and professional assessment (table 3). Most scores included demographic information and triage acuity information as predictors for admission.

\section{General adult population}

We included seven studies that developed or validated a model in the general adult population, aged 18 years and over. $^{18} 1922-2528$ In five studies, in total eight prediction models were developed. ${ }^{19} 22-25$

Discrimination in the derivation cohorts of these newly developed models ranged from AUC (95\% CI) 0.81 (0.790 to 0.820$)$ to $0.878(0.876$ to 0.879$)$. One study did not provide the derivation AUC, but solely provided the AUCs in the validation population. ${ }^{24}$ Four out of five studies also described validation of their developed models. The remaining two studies tested an existing model in their ED. This consisted of the National Early Warning Score ${ }^{28}$ and the GAPS. ${ }^{18}$ Discrimination in the validation studies ranged from AUC $(95 \% \mathrm{CI}) 0.664(0.599$ to 0.728$)$ to 0.876 (0.860 to 0.891$)$. Calibration was described in five studies. Model characteristics are presented in table 4.

\section{Older ED population}

Four studies investigating the older patient population specifically were identified. ${ }^{20212627}$ In these studies, five different models were described, of which three were older patients specific. These models already existed and were used for predicting either frailty or readmission, but not for primary admission. These models included geriatric parameters, such as cognitive impairment and polypharmacy. Discrimination ranged from AUC $(95 \% \mathrm{CI}) 0.63(0.60$ to 0.65$)$ to $0.68(0.66$ to 0.70$)$, which represents poor performance. The other two studies investigated triage systems in older patients. ${ }^{26}{ }^{27}$ The ESI performed best in predicting admission with an AUC $(95 \% \mathrm{CI})$ of $0.74(0.73$ to 0.75$) .^{27}$ None of the models were calibrated, nor tested in external populations in these articles.

One study compared the older patient population with the general adult population. ${ }^{25}$ This study developed and validated an admission model using temporal validation. The model performed slightly worse in the older ED population, but yielded a good AUC (95\% CI) of 0.81 ( 0.79 to $0.82)$, which dropped to $0.77(0.75$ to 0.79$)$ after external validation. The positive predictive value and the positive likelihood ratio were higher in the older population. They concluded that further research is needed to investigate the combination of disease severity with frailty to improve prediction of hospital admission in the older patient population. Model characteristics in the older ED population are presented in table 5 .

\section{DISCUSSION}

The aim of this systematic review was to find and evaluate prediction models for admission used at the ED. We systematically reviewed 11 papers describing the development or validation of 16 different admission prediction models. Selection of the most appropriate model is based on mainly two qualifications: the model with the lowest probability of overall bias and the highest predictive performances for admission. 
Table 3 Categorisation of parameters in the prediction models

\begin{tabular}{|c|c|c|c|c|c|c|c|c|c|c|c|}
\hline & Model & Demographics & $\begin{array}{l}\text { Vital } \\
\text { signs }\end{array}$ & Interventions & Triage & $\begin{array}{l}\text { Previous } \\
\text { care } \\
\text { contacts }\end{array}$ & $\begin{array}{l}\text { Chief } \\
\text { complaint }\end{array}$ & $\begin{array}{l}\text { Drug } \\
\text { use }\end{array}$ & $\begin{array}{l}\text { Mobility and } \\
\text { dependency }\end{array}$ & $\begin{array}{l}\text { ED } \\
\text { entrance }\end{array}$ & $\begin{array}{l}\text { Professional } \\
\text { assessment }\end{array}$ \\
\hline Alam et al ${ }^{28}$ & NEWS & & $x$ & $x$ & & & & & & & \\
\hline $\begin{array}{l}\text { Cameron } \\
\text { et } a l^{19} \text { and } \\
\text { Cameron et } \\
a^{18}\end{array}$ & GAPS & $x$ & $x$ & $x$ & $x$ & $x$ & & & & $x$ & \\
\hline $\begin{array}{l}\text { Di Bari et al }{ }^{20} \\
\text { and Salvi et } \\
a l^{21}\end{array}$ & ISAR & $x$ & & & & $x$ & & $x$ & $x$ & & \\
\hline Di Bari et a $2^{20}$ & SC & $x$ & & & & $x$ & & $x$ & & & \\
\hline $\begin{array}{l}\text { Grossmann } \\
\text { et } a^{27}\end{array}$ & ESI & & & & $\mathrm{x}$ & & & & & & \\
\hline Lucke et $a l^{25}$ & $\begin{array}{l}\text { Older patient } \\
\text { model }\end{array}$ & $x$ & $x$ & $x$ & $x$ & $x$ & $x$ & & & & $x$ \\
\hline Noel et $a l^{22}$ & TNP & & & & & & & & & & $x$ \\
\hline Noel et $a l^{22}$ & Own model & $x$ & & & $x$ & & $x$ & & & $x$ & \\
\hline Noel et $a l^{22}$ & $\begin{array}{l}\text { TNP+own } \\
\text { model }\end{array}$ & $x$ & & & $x$ & & $x$ & & & $x$ & $x$ \\
\hline Salvi et al21 & TRST & & & & & $x$ & & $x$ & $x$ & & $x$ \\
\hline Zlotnik et all ${ }^{23}$ & Own model LR & $x$ & & & $x$ & & $x$ & & & $x$ & \\
\hline Zlotnik et a $\left.\right|^{23}$ & $\begin{array}{l}\text { Own model } \\
\text { ANN }\end{array}$ & $x$ & & & $x$ & & $x$ & & & $x$ & \\
\hline
\end{tabular}

Online supplemental appendix B.

ANN, Artificial Neural Network; AVPU, Alert, Verbal, Pain, Unresponsive; ESI, Emergency Severity Index; GAPS, Glasgow Admission Prediction Score; GP, general practitioner; ISAR, Identification of Seniors At Risk; LR, logistic regression; MTS, Manchester Triage System; NEWS, National Early Warning Score; SC, Silver Code; TNP, Triage Nurse Prediction; TRST,

Triage Risk Screening Tool; VAS, Visual Analogue Scale.

Five models reported an AUC over 0.85. ${ }^{18} 19222325$ The discrimination statistic was highest for the GAPS model. ${ }^{18} 19$

We identified 12 external validations of an admission model. External validation of these models showed substantial variation in performance. This is probably attributable to the fact that some models were tested for a different outcome than they were intended for. Moreover, discrimination may be moderate because ED populations are heterogeneous. Calibration was executed only in 5 of the 11 studies. All models reporting calibration were well-calibrated. ${ }^{1819} 222325$

Apart from the quality of the model, the model should also be easily applicable. In the ED, it is useful if the parameters used can be obtained directly, are objective and are reproducible. Easily obtainable parameters are predictors that can be retrieved at ED entrance. This will enable immediate use of the prediction model. Several models however use parameters that require (collateral) history, which may limit the utility of the prediction model. This information is often not immediately available. The predictors should also be objective, that is, having a low inter-rater and intra-rater variability. Two studies used the judgement of admission of a healthcare professional as a predictor, ${ }^{18} 22$ which is a subjective predictor.

To allow implementation in clinical practice, models should be easy interpretable or provide applications to enable more complex calculations.
We found that several studies did not report key study details, which made it difficult to judge model utility and make external validation impossible. With the arrival of machine learning in medical prediction research, models have become more complex. The benefit of machine learning is that models improve from experience. However, machine learning limits insight of how the prediction model works and also limits external validation.

\section{Strengths and limitations}

This is to our knowledge the first systematic review on prediction models for admission to the hospital from the ED. Strengths of this study include the comprehensive literature search, selection of articles, standard assessment of the articles and the quality assessment using the CHARMS checklist, which was all performed by two researchers separately. However, also several limitations should be considered. We did limit the inclusion of studies to studies executed in European EDs. We possibly excluded non-European models, which could be applicable to the European ED setting. Even despite only selecting European studies, practice and organisation between countries and even between different EDs in the same country are different. Applicability of a prediction tool is dependent on how the healthcare system is organised. Furthermore, the number of included studies might be reduced by only including studies in English. The general 
Table 4 Performance of admission prediction models in the adult population

\begin{tabular}{|c|c|c|c|c|c|c|c|c|}
\hline Study & $\begin{array}{l}\text { Model } \\
\text { name }\end{array}$ & $\begin{array}{l}\text { Admission, } \\
\text { N (\%) }\end{array}$ & Derivation AUC $(95 \% \mathrm{CI})$ & $\begin{array}{l}\text { Calibration } \\
\text { method }\end{array}$ & $\begin{array}{l}\text { Calibration } \\
\text { derivation }\end{array}$ & $\begin{array}{l}\text { Validation } \\
\text { method }\end{array}$ & Validation AUC $(95 \% \mathrm{Cl})$ & $\begin{array}{l}\text { Calibration } \\
\text { validation }\end{array}$ \\
\hline Alam et $\left.a\right|^{28}$ & NEWS & $130(47.4)$ & & & & External & $\begin{array}{l}\text { t0: } 0.664(0.599 \text { to } 0.728) \text { t1: } \\
0.687(0.620 \text { to } 0.754) \text { t2: } \\
0.697(0.609 \text { to } 0.786)\end{array}$ & \\
\hline Cameron et al ${ }^{19}$ & GAPS & NS & $0.8778(0.8764$ to 0.8793$)$ & HL GOF test & & Split sample & 0.8774 (0.8752 to 0.8796$)$ & $p=0.524$ \\
\hline Cameron et al ${ }^{18}$ & GAPS & $745(40.7)$ & & $\begin{array}{l}\text { Wilcoxon } \\
\text { Signed Rank } \\
\text { test }\end{array}$ & & External & 0.876 (0.860 to 0.892$)$ & $1.20 \%$ \\
\hline Cameron et al ${ }^{18}$ & VAS & $745(40.7)$ & & $\begin{array}{l}\text { Wilcoxon } \\
\text { Signed Rank } \\
\text { test }\end{array}$ & & External & 0.875 (0.859 to 0.891$)$ & $9.20 \%$ \\
\hline $\begin{array}{l}\text { Kraaijvanger } \\
\text { et } a l^{24}\end{array}$ & Own model & $400(31.7)$ & NS & $\begin{array}{l}\text { Calibration } \\
\text { plot }\end{array}$ & & External & $\begin{array}{ll}\text { 1. } & 0.88(0.85 \text { to } 0.90), \\
\text { 2. } & 0.87(0.85 \text { to } 0.89) \text {, } \\
\text { 3. } & 0.76(0.72 \text { to } 0.80)\end{array}$ & $\begin{array}{ll}\text { 1. } & \alpha: 0.023, \beta: \\
& 0.974 \\
\text { 2. } & \alpha: 0.05, \beta: \\
& 0.98\end{array}$ \\
\hline Lucke et $a l^{25}$ & $\begin{array}{l}\text { Own model } \\
\text { adults }\end{array}$ & $4044(23.6)$ & $0.85(0.84$ to 0.86$)$ & $\begin{array}{l}\text { Calibration } \\
\text { plot, HL GOF } \\
\text { test }\end{array}$ & & External & $0.86(0.85$ to 0.87$)$ & $p>0.05$ \\
\hline Noel et $a l^{22}$ & TNP & $2313(23.5)$ & $0.815(0.805$ to 0.826$)$ & & & & & \\
\hline Noel et $a l^{22}$ & Own model & $2313(23.5)$ & $0.815(0.805$ to 0.825$)$ & & & & & \\
\hline Noel et $a l^{22}$ & $\begin{array}{l}\text { TNP+own } \\
\text { model }\end{array}$ & $2313(23.5)$ & 0.857 (0.848 to 0.865$)$ & & & & & \\
\hline Zlotnik et $a l^{23}$ & $\begin{array}{l}\text { Own model } \\
\text { LR }\end{array}$ & 34694 (13.6) & $0.8611(0.8568$ to 0.8615$)$ & $\begin{array}{l}\text { Calibration } \\
\text { plot, HL GOF } \\
\text { test }\end{array}$ & $\chi^{2}=85.18$ & Split sample & 0.8568 (0.8508 to 0.8583$)$ & $\chi^{2}=65.32$ \\
\hline Zlotnik et $\left.a\right|^{23}$ & $\begin{array}{l}\text { Own model } \\
\text { ANN }\end{array}$ & $34694(13.6)$ & $0.8631(0.8605$ to 0.8656$)$ & $\begin{array}{l}\text { Calibration } \\
\text { plot, HL GOF } \\
\text { test }\end{array}$ & $\chi^{2}=16.01$ & Split sample & $0.8575(0.8540$ to 0.8610$)$ & $\chi^{2}=17.28$ \\
\hline
\end{tabular}

Empty cells mean that specific characteristics were not tested.

$\alpha$, calibration intercept; $\beta$, calibration slope; ANN, artificial neural network; AUC, area under the curve; GAPS, Glasgow Admission Prediction Score; HL GOF, Hosmer-Lemeshow goodness of fit; LR, logistic regression; N, number; NEWS, National Early Warning Score; NS, not specified; t, timepoint; TNP, triage nurse prediction; VAS, Visual Analogue Scale.;

limitation of reporting prediction models for admission in the ED is the heterogeneity in ED patients, which is due to epidemiological differences in the populations. This makes it difficult to compare prediction models and to combine these studies in a meta-analysis.

\section{Future directions}

None of the studies described implementation, and to our knowledge, none of the models are currently implemented in the ED as a prediction tool for admission. The lack of implementation cannot be explained by the discriminative ability, which was generally good. Model calibration was lacking in most studies, and therefore, it is difficult to judge whether a model, which performs well at group level, is also performing well for individual patients.

Future research should focus on validation, utility of additional predictors, exploration of electronic implementation in patient files to enable the clinical use of prediction models and analysis of their impact. Currently, impact analysis in prediction research is sparse, making it difficult to conclude whether a model is worth implementing as an adjunct to clinical evaluation. In the ED, it is worthwhile to investigate whether implementation of an admission prediction model reduces ED crowding and improves patient outcomes in terms of a shorter LOS at the ED and in the hospital.

Table 5 Performance of admission prediction models in the older population

\begin{tabular}{|c|c|c|c|c|c|c|c|}
\hline Study & Model name & Admission, N (\%) & $\begin{array}{l}\text { Derivation AUC } \\
(95 \% \mathrm{Cl})\end{array}$ & Validation method & Validation AUC & Calibration method & Calibration \\
\hline Brouns et $a l^{26}$ & MTS & $4223(59.4)$ & & External & $0.74(0.73-0.75)$ & & \\
\hline Di Bari et a $l^{20}$ & ISAR & $558(34)$ & & External & $0.65(0.62-0.68)$ & & \\
\hline Grossmann et al ${ }^{27}$ & ESI & $250(48.8)$ & & External & $0.741(0.734-0.747)$ & & \\
\hline Lucke et $a l^{25}$ & $\begin{array}{l}\text { Own model older } \\
\text { patients }\end{array}$ & $1817(43.8)$ & 0.81 (0.79 to 0.82 ) & External & $0.77(0.75-0.79)$ & $\begin{array}{l}\text { Calibration plot, } \\
\text { GOF test }\end{array}$ & $p>0.05$ \\
\hline Salvi et $a l^{21}$ & TRST & $626(30)$ & & External & $0.66(0.64-0.69)$ & & \\
\hline
\end{tabular}

Empty cells mean that specific characteristics were not tested.

AUC, area under the curve; ESI, emergency severity index; GOF, goodness of fit; ISAR, identification of seniors at risk; MTS, Manchester triage system; SC, silver code; TRST, triage risk screening tool. 
Furthermore, we recommend that models should be validated and updated to judge generalisability to specific populations prior to implementation. We also recommend that with every external validation study, calibration should be reported.

\section{CONCLUSION}

This systematic review identified 16 prognostic models for predicting admission in patients presenting to the ED. The models of Cameron and Lucke were well developed and have adequate predictive performance. We suggest that the effect of these models on ED LOS and crowding reduction should be examined, given that external validation and potentially updating of the models have taken place for the specific hospital ED.

Contributors Conceptualisation: $A B, J A$, SS; study identification: WMB; study selection: $A B$, LAAMvA, JA; data extraction: $A B$, LAAMvA; methodology: $A B, J A, H L$, SS; supervision: RZ, HL, JA, SS; writing —original draft: $A B$, LAAMvA; writing — review and editing: JA, HL, RZ, SS, WMB.

Funding The authors have not declared a specific grant for this research from any funding agency in the public, commercial or not-for-profit sectors.

Competing interests None declared.

Patient consent for publication Not applicable.

Provenance and peer review Not commissioned; externally peer reviewed.

Open access This is an open access article distributed in accordance with the Creative Commons Attribution 4.0 Unported (CC BY 4.0) license, which permits others to copy, redistribute, remix, transform and build upon this work for any purpose, provided the original work is properly cited, a link to the licence is given, and indication of whether changes were made. See: https://creativecommons.org/ licenses/by/4.0/

ORCID iDs

Anniek Brink http://orcid.org/0000-0001-9072-2469

Jelmer Alsma http://orcid.org/0000-0002-2808-1514

\section{REFERENCES}

1 Crowding. Ann Emerg Med 2019;74:e31.

2 Bernstein SL, Aronsky D, Duseja R, et al. The effect of emergency department crowding on clinically oriented outcomes. Acad Emerg Med 2009;16:1-10.

3 Epstein SK, Huckins DS, Liu SW, et al. Emergency department crowding and risk of preventable medical errors. Intern Emerg Med 2012;7:173-80.

4 Salehi L, Phalpher P, Valani R, et al. Emergency department boarding: a descriptive analysis and measurement of impact on outcomes. CJEM 2018:20:929-37.

5 Sun BC, Hsia RY, Weiss RE, et al. Effect of emergency department crowding on outcomes of admitted patients. Ann Emerg Med 2013;61:605-11.

6 Jo S, Jeong T, Jin YH, et al. Ed crowding is associated with inpatient mortality among critically ill patients admitted via the ED: post hoc analysis from a retrospective study. Am J Emerg Med 2015;33:1725-31.
7 Mataloni F, Pinnarelli L, Perucci CA, et al. Characteristics of ED crowding in the Lazio region (Italy) and short-term health outcomes. Intern Emerg Med 2019;14:109-17.

8 Asplin BR, Magid DJ, Rhodes KV, et al. A conceptual model of emergency department crowding. Ann Emerg Med 2003:42:173-80.

9 Lowthian JA, Curtis AJ, Cameron PA, et al. Systematic review of trends in emergency department attendances: an Australian perspective. Emerg Med J 2011;28:373-7.

10 Aminzadeh F, Dalziel WB. Older adults in the emergency department: a systematic review of patterns of use, adverse outcomes, and effectiveness of interventions. Ann Emerg Med 2002;39:238-47.

11 Paton A, Mitra B, Considine J. Longer time to transfer from the emergency department after bed Request is associated with worse outcomes. Emerg Med Australas 2019;31:211-5.

12 Ebker-White A, Bein KJ, Berendsen Russell S, et al. The Sydney triage to admission risk tool (start) to improve patient flow in an emergency department: a model of care implementation pilot study. BMC Emerg Med 2019;19:79.

13 Moher D, Liberati A, Tetzlaff J, et al. Preferred reporting items for systematic reviews and meta-analyses: the PRISMA statement. BMJ 2009;339:b2535.

14 Bramer WM, Giustini D, de Jonge GB, et al. De-duplication of database search results for systematic reviews in endnote. J Med Libr Assoc 2016;104:240-3.

15 Moons KGM, de Groot JAH, Bouwmeester W, et al. Critical appraisal and data extraction for systematic reviews of prediction modelling studies: the charms checklist. PLoS Med 2014:11:e1001744.

16 Pencina MJ, D'Agostino RB. Evaluating discrimination of risk prediction models: the C statistic. JAMA 2015:314:1063-4.

17 Steyerberg EW, Vickers AJ, Cook NR, et al. Assessing the performance of prediction models: a framework for traditional and novel measures. Epidemiology 2010;21:128-38

18 Cameron A, Ireland AJ, McKay GA, et al. Predicting admission at triage: are nurses better than a simple objective score? Emerg Med J 2017;34:2-7.

19 Cameron A, Rodgers K, Ireland A, et al. A simple tool to predict admission at the time of triage. Emerg Med J 2015;32:174-9.

20 Di Bari M, Salvi F, Roberts AT, et al. Prognostic stratification of elderly patients in the emergency department: a comparison between the "Identification of Seniors at Risk" and the "Silver Code". J Gerontol A Biol Sci Med Sci 2012;67:544-50.

21 Salvi F, Morichi V, Lorenzetti B, et al. Risk stratification of older patients in the emergency department: comparison between the identification of seniors at risk and triage risk screening tool. Rejuvenation Res 2012;15:288-94.

22 Noel G, Bonte N, Persico N, et al. Real-time estimation of inpatient beds required in emergency departments. Eur J Emerg Med 2019;26:440-5.

23 Zlotnik A, Alfaro MC, Pérez MCP, et al. Building a decision support system for inpatient admission prediction with the Manchester triage system and administrative Check-in variables. Comput Inform Nurs 2016:34:224-30.

24 Kraaijvanger N, Rijpsma D, Roovers $L$, et al. Development and validation of an admission prediction tool for emergency departments in the Netherlands. Emerg Med J 2018:35:464-70

25 Lucke JA, de Gelder J, Clarijs F, et al. Early prediction of hospital admission for emergency department patients: a comparison between patients younger or older than 70 years. Emerg Med J 2018:35:18-27.

26 Brouns SHA, Mignot-Evers L, Derkx F, et al. Performance of the Manchester triage system in older emergency department patients: a retrospective cohort study. BMC Emerg Med 2019;19:3.

27 Grossmann FF, Zumbrunn T, Frauchiger A, et al. At risk of undertriage? testing the performance and accuracy of the emergency severity index in older emergency department patients. Ann Emerg Med 2012;60:317-25.

28 Alam N, Vegting IL, Houben E, et al. Exploring the performance of the National early warning score (news) in a European emergency department. Resuscitation 2015;90:111-5. 